\section{Tratamiento ortodóntico con extracción de un incisivo inferior. Reporte de un caso}

\section{Orthodontic treatment with one lower incisor extraction. A case report}

\section{Resumen}

Mujer de 22 años de edad con biotipo mesofacial, patrón esquelético de Clase I y buen perfil de labios, presentó una maloclusión de Angle de Clase I con buena intercuspidación de molares y premolares, con apińamiento moderado superior e inferior, discrepancia de Bolton anterior por exceso inferior y overbite y overjet disminuidos. Se realizó un tratamiento de ortodoncia con la extracción de un incisivo inferior, una de las extracciones atípicas en ortodoncia. El éxito de este tipo de tratamiento requiere de un profundo y minucioso registro diagnóstico en ortodoncia, ya que su elección dependerá de que el caso clínico reúna una serie de requisitos.

Palabras clave: Extracción dental; Incisivo; Ortodoncia; Tratamiento.

\begin{abstract}
A 22 year old woman with mesofacial biotype, skeletal class I and good lips profile, presented an Angle malocclusion class I with good intercuspidation of molars and premolars, moderate crowding in both arches, discrepancy of anterior Bolton for inferior excess, and decreased overjet and overbite. She was treated with a orthodontics treatment with extraction of a lower incisor, an atypical extractions in orthodontics. The success of this type of treatment needs a deep and meticulous diagnostic record, because this election of treatment will depend on a series of minimum requirements.
\end{abstract}

Keywords: Incisor; Orthodontics; Therapeutics; Tooth extraction.

\section{Caso Clínico}

Luis Carlos Vélez Taboada ${ }^{1 a}$, Manuel Gustavo Chávez Sevillano ${ }^{1 b}$, Hugo Javier Luque Luque ${ }^{1 b}$

${ }^{1}$ Facultad de Odontología. Universidad Nacional Mayor de San Marcos. Lima, Perú.

a Cirujano Dentista.

${ }^{\mathrm{b}}$ Especialista en Ortodoncia y Ortopedia Maxilar.

Correspondencia:

Luis Carlos Vélez Taboada

Correo electrónico: luis_carlos_velez@hotmail. com

Av. Ramón Herrera 343, Urb. Benavides. Lima 1, Perú.

\section{Coautores:}

Manuel Gustavo Chávez Sevillano

mchavezs@unmsm.edu.pe

Hugo Javier Luque Luque

hjll50@hotmail.com

Conflicto de intereses: Los autores declaran no tener conflictos de interés.

Fuente de financiamiento: Autofinanciado.

Fecha de recepción: 21/09/17

Fecha de aceptación: 09/10/17 


\section{Introducción}

Las extracciones dentales con motivos ortodónticos es una práctica frecuente en el tratamiento de las maloclusiones dentarias. Los premolares son los dientes más frecuentemente indicados para extracciones en ortodoncia, cuando el objetivo es conseguir espacio en la arcada para eliminar o reducir el apiñamiento, mejorar el perfil de los labios y/o corregir asimetrías ${ }^{1}$. En la gran mayoría de los casos el apiñamiento se encuentra concentrado en la zona de incisivos y caninos, y debido a que los premolares son los dientes más cercanos a la zona del apiñamiento, estos son los preferidos y la opción más común al momento de elegir las extracciones. Sin embargo, se ha planteado también la posibilidad de extracción de un incisivo inferior como plan de tratamiento en ortodoncia ${ }^{2}$.

Bahreman ${ }^{3}$ presentó la extracción de un incisivo inferior como una opción terapéutica para el tratamiento de ortodoncia en algunos casos específicos. La extracción de un incisivo inferior en el tratamiento de ortodoncia es poco frecuente, de 1,1 a $6 \%$ de todos los tratamientos con extracciones ${ }^{4}$.

El tratamiento de ortodoncia con extracción de un incisivo mandibular está indicado para pacientes sin crecimiento, cuando el objetivo de tratamiento es mantener el perfil de los labios, y cuando se requiere que sea de corta duración a pesar de ser extractivo ${ }^{4-11}$.

Los requisitos intraorales para la extracción de un incisivo son: (1) maloclusión dentaria de Clase I con relaciones caninas de Clase I y adecuada intercuspidación posterior, (2) discrepancia alveolo-dentaria moderada y/o severa en sector anteroinferior $>5 \mathrm{~mm}$, (3) Bolton anterior por exceso de masa inferior $>4 \mathrm{~mm}$, (4) overbite y overjet disminuidos. Como es observado también en maloclusiones esqueléticas de Clase III leves o moderadas, con relaciones molares de Clase I de Angle, y (5) adicionalmente favorece tener incisivos inferiores con corona rectangular e incisivos superiores de forma triangular $^{4-11}$.

Las contraindicaciones para un tratamiento con la extracción de un incisivo son: (1) overbite y overjet aumentado, (2) apińamiento superior moderado o severo de incisivos y caninos, (3) incisivos superiores que requieran aumento de su ancho mesiodistal debido a anomalías de forma y tamaño, (4) enfermedad periodontal del grupo de incisivos y caninos inferiores, y (5) presencia de triángulos negros ${ }^{4-11}$.

La decisión de cuál incisivo inferior extraer va a depender del estado de salud estructural y periodontal de cada uno de ellos, como por ejemplo, dientes restaurados, traumatizados o con endodoncia, recesión gingival, dehiscencia ósea y/o con erupción normal o ectópica ${ }^{4-14}$.

La principal recomendación en la planificación de un tratamiento de ortodoncia con extracción de un incisivo inferior es la realización previa de un Setup de modelos ortodóncicos. Con el Setup es posible predecir el resultado final del tratamiento, como el overbite, overjet, el cierre de espacios de la extracción, y la necesidad de desgaste interproximal superior dentro los límites permitidos $^{4-12}$.

Como resultado del tratamiento de ortodoncia con extracción de un incisivo: (1) tenemos un exceso de Bolton antero superior, que se resuelve generalmente con desgaste interproximal superior, (2) incremento del overbite y overjet, (3) la línea media dentaria superior debe coincidir con la mitad del ancho mesiodistal del incisivo de posición central de la arcada inferior, y (4) disminución del ancho intercanino y tendencia a la inclinación y migración hacia mesial ${ }^{4-15}$.

Aunque las alternativas de tratamiento para resolver el apiñamiento son variadas, se reporta el caso clínico tratado con aparatología fija ortodóncica con extracción de un incisivo central inferior.

\section{Reporte del caso}

Diagnóstico y etiología. Mujer de 22 años 11 meses acudió a la Clínica de Posgrado de la Facultad de Odontología de la Universidad Nacional Mayor de San Marcos (UNMSM) - Lima, Perú. Como motivo de consulta la paciente refirió querer hacerse el tratamiento de ortodoncia por la presencia de apiñamiento dental.

El análisis facial inicial indicó un tipo facial mesofacial (Índice morfológico facial $=0,9$ ), un perfil total recto y buen perfil de labios en reposo (Figura 1). El examen clínico intraoral y análisis de modelos determinó una maloclusión dentaria de Clase I de Angle con una buena intercuspidación de molares y premolares, apiñamiento anterior superior e inferior de $-2,5 \mathrm{~mm}$ y $-4 \mathrm{~mm}$ respectivamente, discrepancia de Bolton anterior por exceso inferior de 3,5 mm, y overbite y overjet disminuidos: $12 \%$ y $1 \mathrm{~mm}$ respectivamente (Figuras 1 y 2 ).

La radiografía panorámica pretratamiento mostró la presencia de cuatro terceras molares no erupcionadas y la dilaceración radicular leve hacia mesial de la pieza 12 (Figura 3). El análisis de la radiografía cefalométrica indicó una relación esquelética de Clase $\mathrm{I}\left(\mathrm{ANB}=1^{\circ}\right)$, patrón de crecimiento facial normodivergente (Eje $\mathrm{Y}=$ $57^{\circ}$ ), plano mandibular con inclinación normodivergente $\left(\mathrm{FMA}=22^{\circ}\right)$, incisivo superior protruido y vestibularizado $\left(1: \mathrm{NA}=6,5 \mathrm{~mm} ; 1: \mathrm{NA}=27^{\circ}\right)$ e incisivo inferior vestibularizado $\left(\mathrm{IMPA}=97^{\circ}\right)($ Figura 4 , Tabla). 

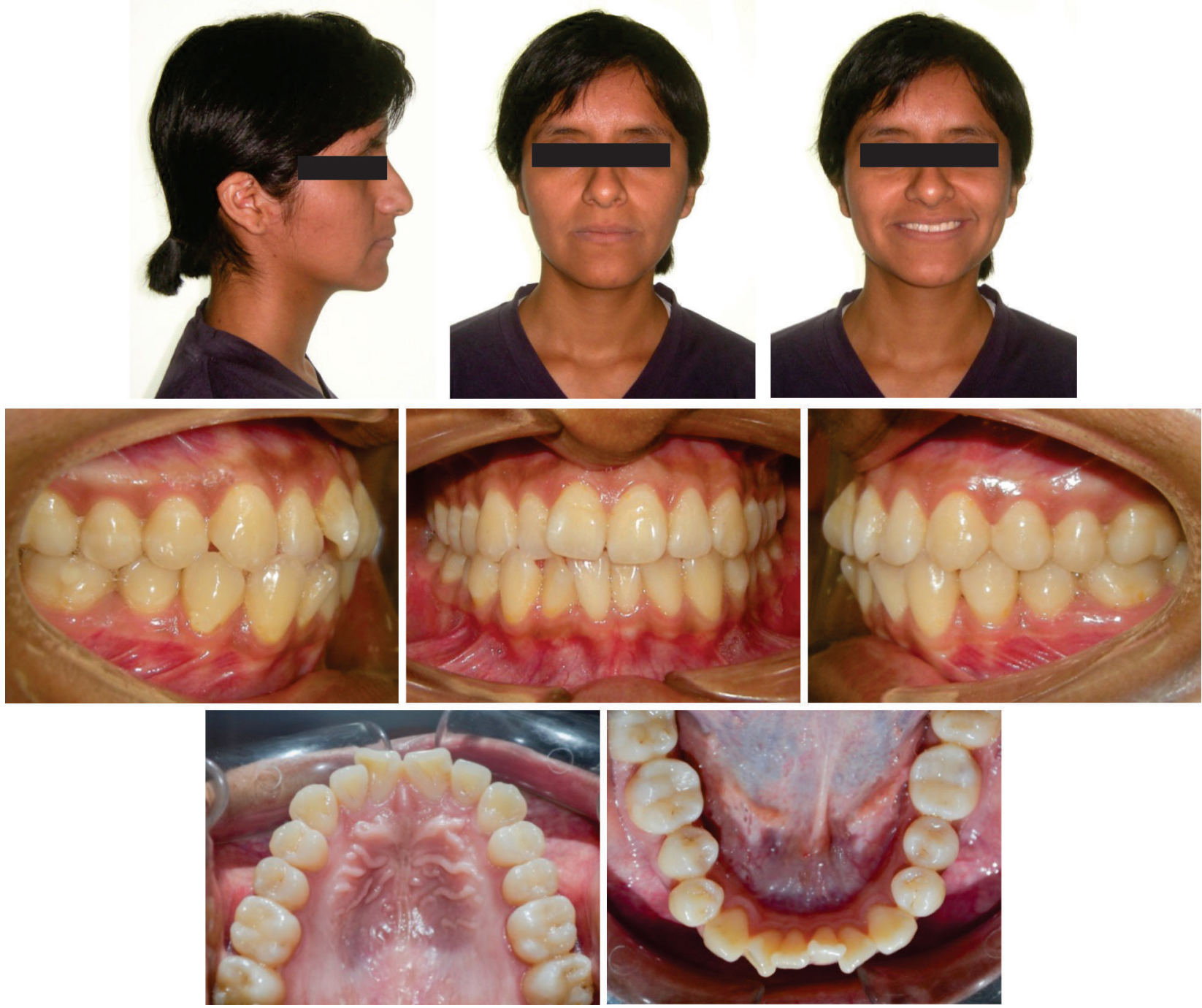

Figura 1. Fotografías extraorales e intraorales pretratamiento.

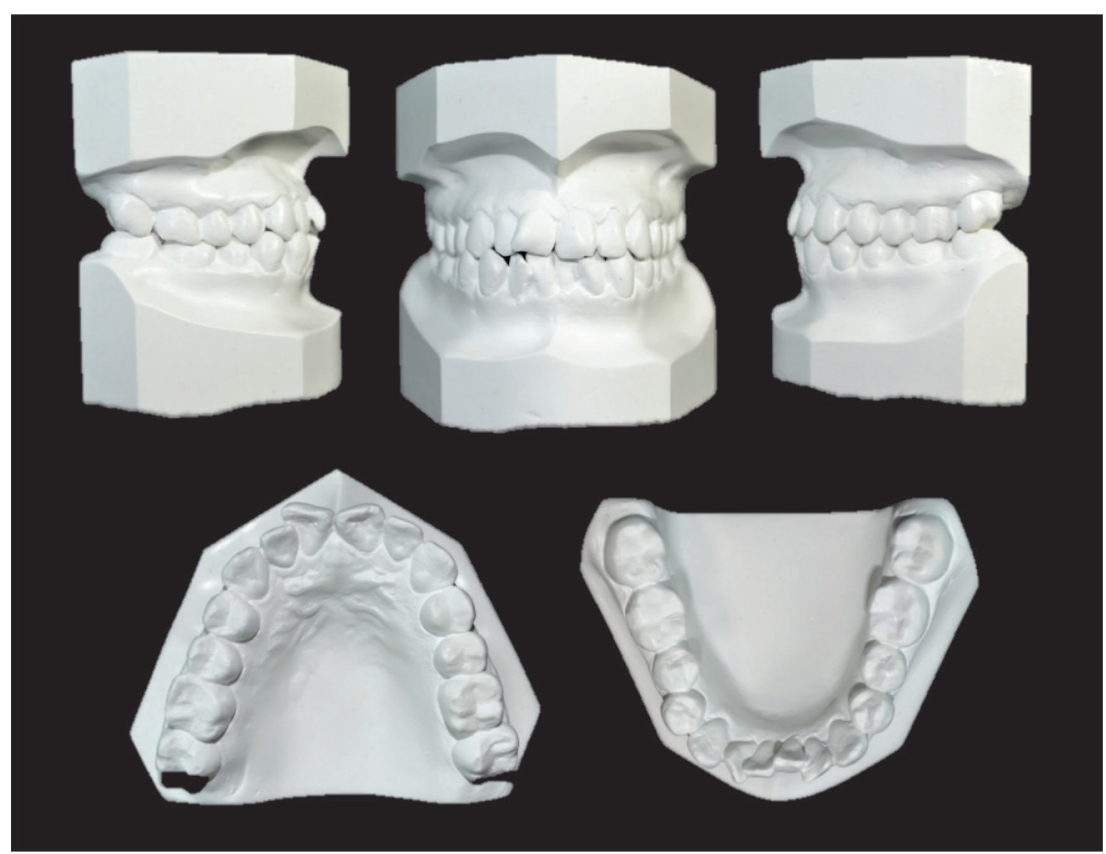

Figura 2. Modelos dentales pretratamiento. 


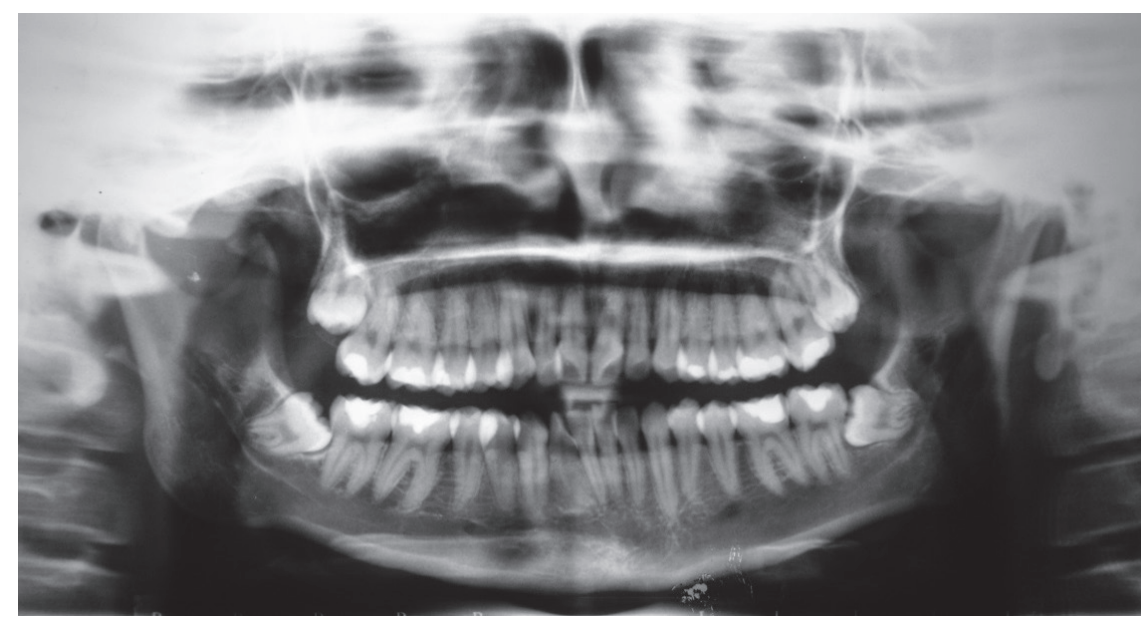

Figura 3. Radiografía panorámica pretratamiento.
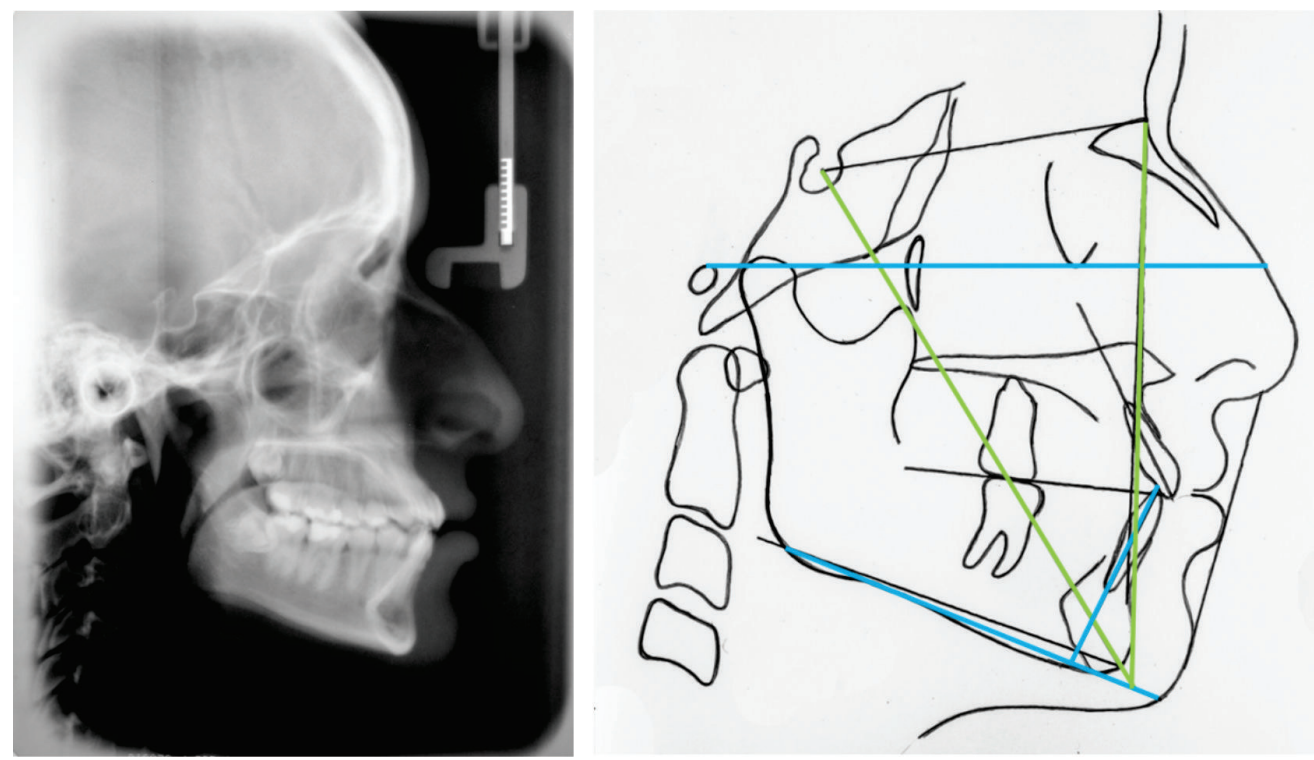

Figura 4. A. Radiografía cefalométrica pretratamiento. B. Trazado cefalométrico pretratamiento. Las líneas de color negro, azul y verde indican el análisis de Steiner, Tweed y Downs respectivamente.

Tabla. Medidas cefalométricas pretratamiento y postratamiento

\begin{tabular}{lll}
\hline & Pretratamiento & Postratamiento \\
\hline SNA $\left(^{\circ}\right)$ & 79 & 78 \\
SNB $\left(^{\circ}\right)$ & 78 & 77 \\
ANB $\left(^{\circ}\right)$ & 1 & 1 \\
SND $\left({ }^{\circ}\right)$ & 76 & 75 \\
1:NA $(\mathrm{mm})$ & 6,5 & 7,5 \\
1:NA $\left(^{\circ}\right)$ & 27 & 33 \\
1:NB $(\mathrm{mm})$ & 5 & 5 \\
1:NB $\left(^{\circ}\right)$ & 27 & 28 \\
Pog:NB $(\mathrm{mm})$ & 1 & 1 \\
IMPA $\left(^{\circ}\right)$ & 97 & 98 \\
FMA $\left({ }^{\circ}\right)$ & 22 & 23 \\
1:1 $\left({ }^{\circ}\right)$ & 128 & 121 \\
Ocl:SN $\left({ }^{\circ}\right)$ & 15 & 20 \\
GoGn:SN $\left(^{\circ}\right)$ & 29 & 29 \\
Labio superior: línea S $(\mathrm{mm})$ & -3 & -3 \\
Labio inferior: línea S $(\mathrm{mm})$ & 0 & -2 \\
Eje Y $\left({ }^{\circ}\right)$ & 58 & 57 \\
\hline
\end{tabular}

Objetivos de tratamiento. Basados en los registros diagnósticos, los objetivos de tratamiento fueron los siguientes: (1) mantener el perfil de labios, (2) mantener la relaciones dentales de Clase I de Angle, (3) corregir el overbite y overjet, (4) eliminar el apiñamiento superior e inferior, (5) eliminar la discrepancia de Bolton y (6) conseguir adecuadas relaciones funcionales de guía anterior y desoclusión caninas derecha e izquierda.

Alternativas de tratamiento. Como primera alternativa de tratamiento se consideró llevar a cabo las extracciones de los cuatro terceros molares y cuatro primeros premolares, ya que este enfoque resolvería de forma efectiva el apińamiento superior e inferior; sin embargo era posible cambiar la posición inicial de los labios hacia una posición más posterior, alterando el buen perfil de los labios. La segunda opción fue la realización de extracciones de los cuatro terceros molares y un incisivo inferior, y desgaste interproximal de incisivos superiores; un enfoque más conservador que evita alterar el perfil de labios en reposo (Figura 5). Se realizó previamente un Setup de 
modelos como parte de la planificación del tratamiento. El paciente escogió la segunda alternativa. El paciente fue informado de los posibles efectos secundarios de un tratamiento de ortodoncia con extracción de un incisivo.

Progreso del tratamiento. Para iniciar el tratamiento de ortodoncia, se llevaron a cabo previamente las exodoncias de los terceros molares. Se instaló un sistema de brackets Edgewise Standard en slot 0,022" (Orthoclas- sic) y se realizó la extracción del incisivo central inferior derecho (pieza 41). Una semana después de la última extracción, se colocó un arco multiloop en acero inoxidable de 0,014 " y un arco continuo de niquel-titanio de 0,014 " en la arcada superior e inferior respectivamente. En el segundo control, se colocó en la arcada superior un arco multiloop en acero inoxidable 0,016 " y en la arcada inferior un arco multiloop en acero inoxidable 0,014" con un box loop para la pieza 42 (Figura 6).

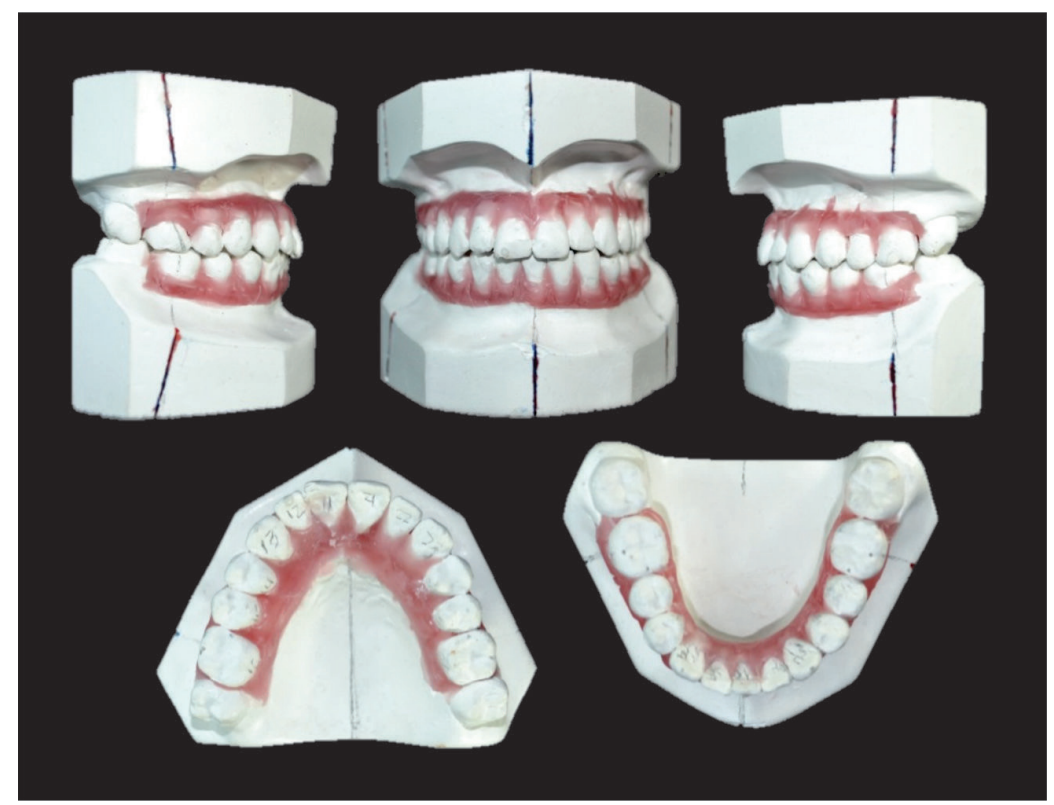

Figura 5. Setup de modelos dentales de tratamiento.
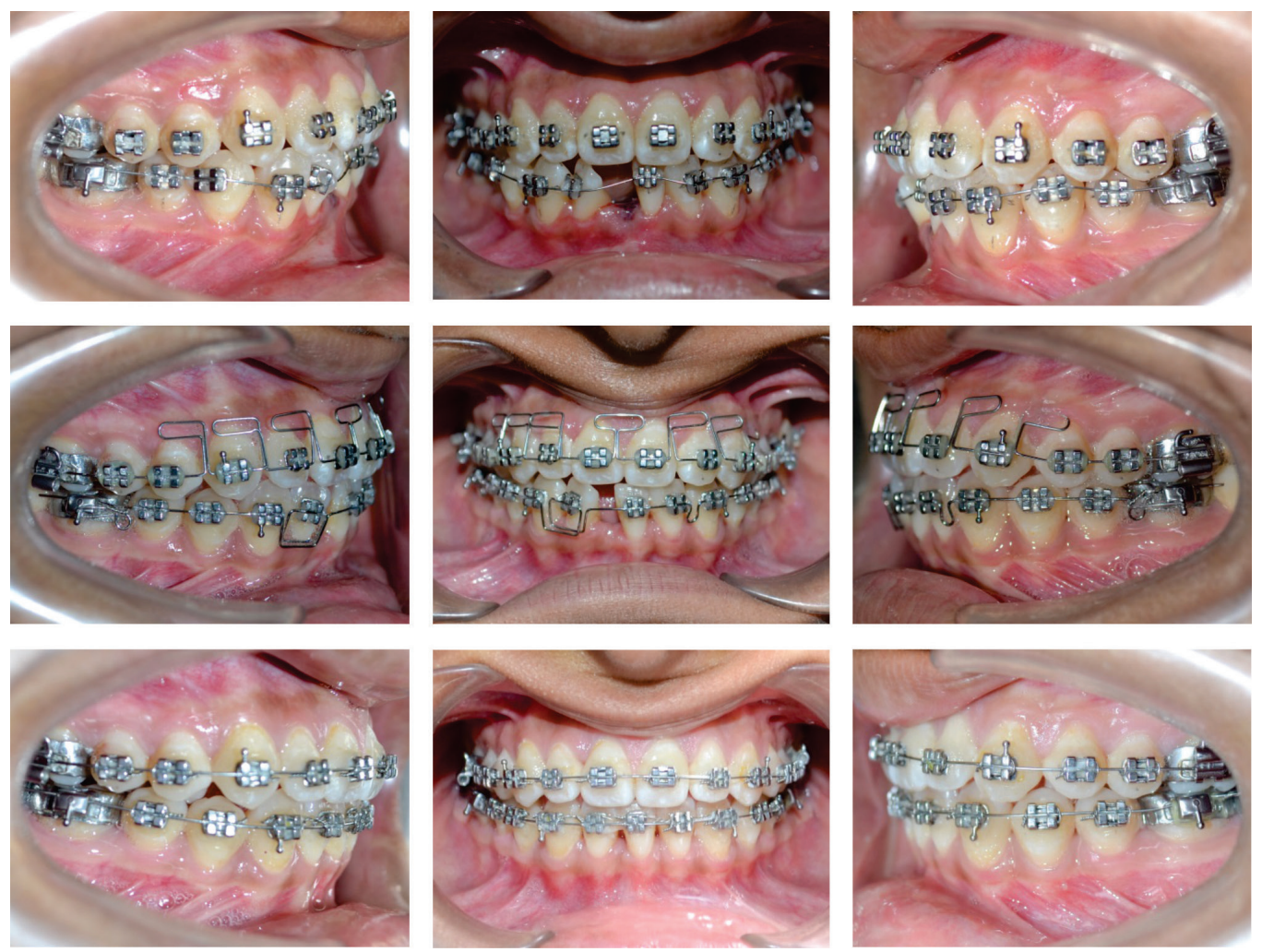

Figura 6. Fotografías intraorales durante las etapas de alineamiento, nivelación y cierre de espacios. 
En las citas sucesivas, en la arcada superior se colocaron arcos continuos en niquel-titanio de 0,016", $0,016 x 0,022$ " y arco en acero inoxidable de 0,018 " con dobleces de primer orden. En la arcada inferior se colocaron arcos continuos en acero inoxidable de 0,014 ", 0,016 " y 0,018 " con dobleces de primer orden. El cierre de espacios en la arcada inferior fue por lingualización de los incisivos remanentes y pérdida de anclaje pasivo, principalmente del lado derecho, que generó una relación molar de Clase III leve (Figura 6).

La última fase del tratamiento, la finalización, (Figura 7) inicia con la colocación de dos arcos multiloop edgewi- se arch wire (MEAW) en niquel-cobalto (Elgiloy azul, RMO) de $0,017 \times 0,025$ " en la arcada superior e inferior con el objetivo de conseguir un buen engranaje posterior de Clase I, usando elásticos intermaxilares de Clase III 3/16 x 6,5onz (Morelli). Nuevamente se regresó a arcos continuos superior e inferior en acero inoxidable de $0,018 \times 0,025$ " con dobleces de primer y tercer orden. Para mejorar aún más la intercuspidación posterior, se realizaron desgastes interproximales de $1 \mathrm{~mm}$ por lado entre las piezas 36/35 y 45/46, que generaron espacios que fueron cerrados después con elásticos intermaxilares de Clase II 3/16 x 4,5 onz (Morelli).
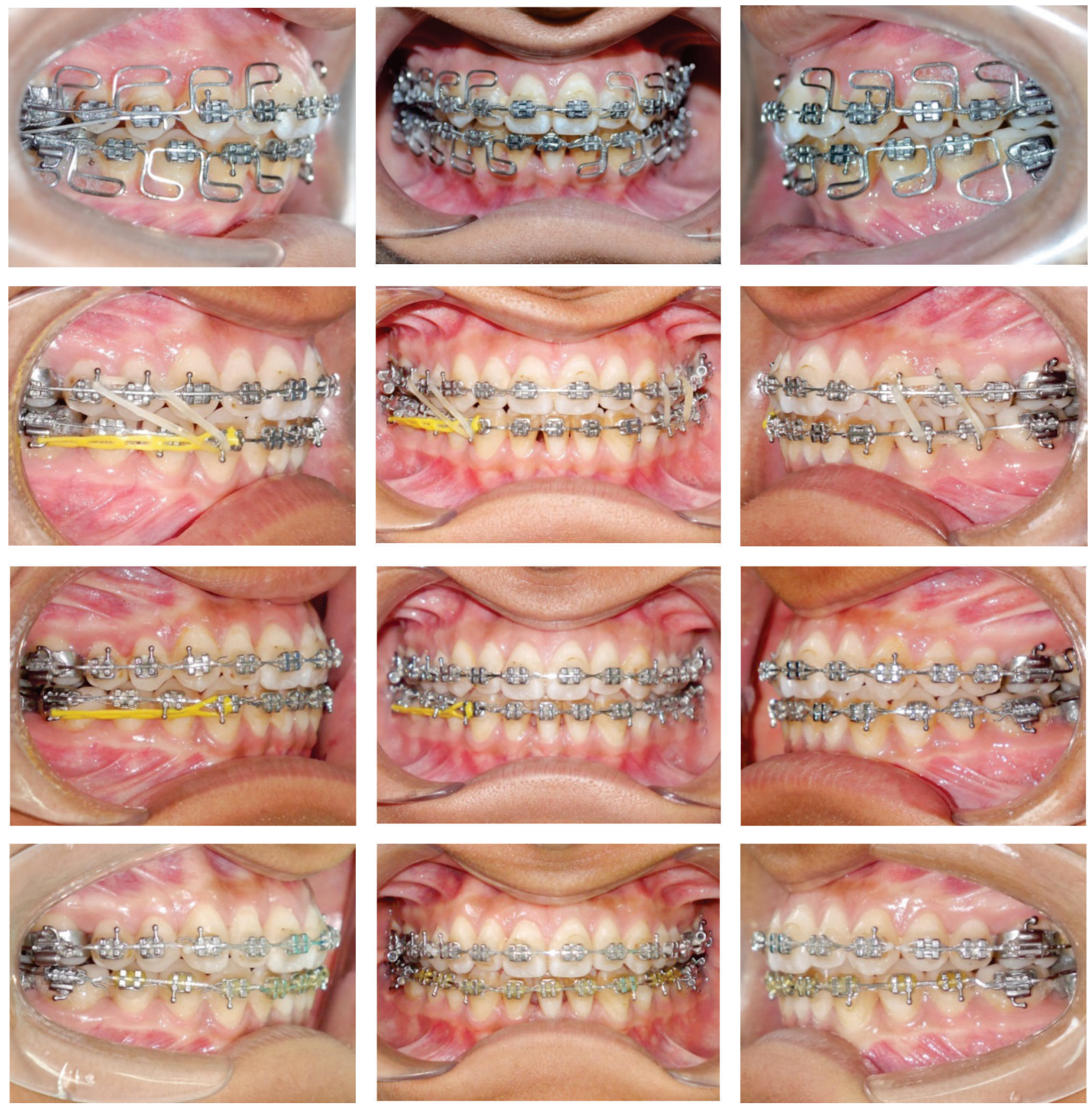

Figura 7. Fotografías intraorales durante la etapa de finalización. 
Para la nivelación de crestas en la arcada inferior se realizaron dobleces de segundo orden: step down de las piezas 36 y 46 en el mismo arco en acero inoxidable de $0,018 \times 0,025$ ".

Para conseguir la guía y acople anterior se realizaron desgastes interproximales de $1,5 \mathrm{~mm}$ en incisivos superiores, que generaron espacios que fueron cerrados progresivamente con alambre de ligadura metálica, ligando en tie together a todos los dientes superiores.

Para conseguir la desoclusión canina derecha, en primer lugar se realizaron en el arco superior en acero inoxidable de $0,018 \times 0,025$ " un doblez de segundo orden: tip forward de corona (pieza 13); y en el arco inferior en acero inoxidable de $0,018 \times 0,025$ " un doblez de segundo orden: tip back de corona (pieza 43). Ambos procedimientos no fueron suficientes. Finalmente, se realizó una remodelación por adicción de la corona para mejorar su anatomía, con el sistema de resina universal Filtek Z350 XT de 3M ESPE.

Tres semanas antes de dar de alta del tratamiento de ortodoncia, se segmentó el arco superior de canino a ca- nino y se le indicó el uso de elásticos intermaxilares de Clase I 3/16 x 4,5onz (Morelli) de canino superior a canino y primer premolar inferior.

El día del retiro de la aparatología fija superior e inferior, se realizó también un pulido y acabado de los todos los dientes y se tomaron impresiones. Al día siguiente se instalaron una contención superior removible confeccionada con alambre y acrílico, y la contención inferior fija de canino a canino en acero inoxidable de $0,017 \times 0,025$ ”.

Resultados del tratamiento. Después de 25 meses de tratamiento, se mantuvieron las relaciones molares, premolares y caninos de Clase I derecha e izquierda, se corrigió el overbite y overjet, se eliminó el apiñamiento superior e inferior, se eliminó la discrepancia de Bolton y se consiguió que la línea media dental superior coincida con la mitad del ancho mesiodistal de la pieza 31 (Figuras 8 y 9). En oclusión dinámica se consiguieron adecuadas relaciones funcionales de guía anterior y desoclusión canina derecha e izquierda.

La evaluación de la radiografía panorámica muestra mínima reabsorción radicular en los ápices de incisivos
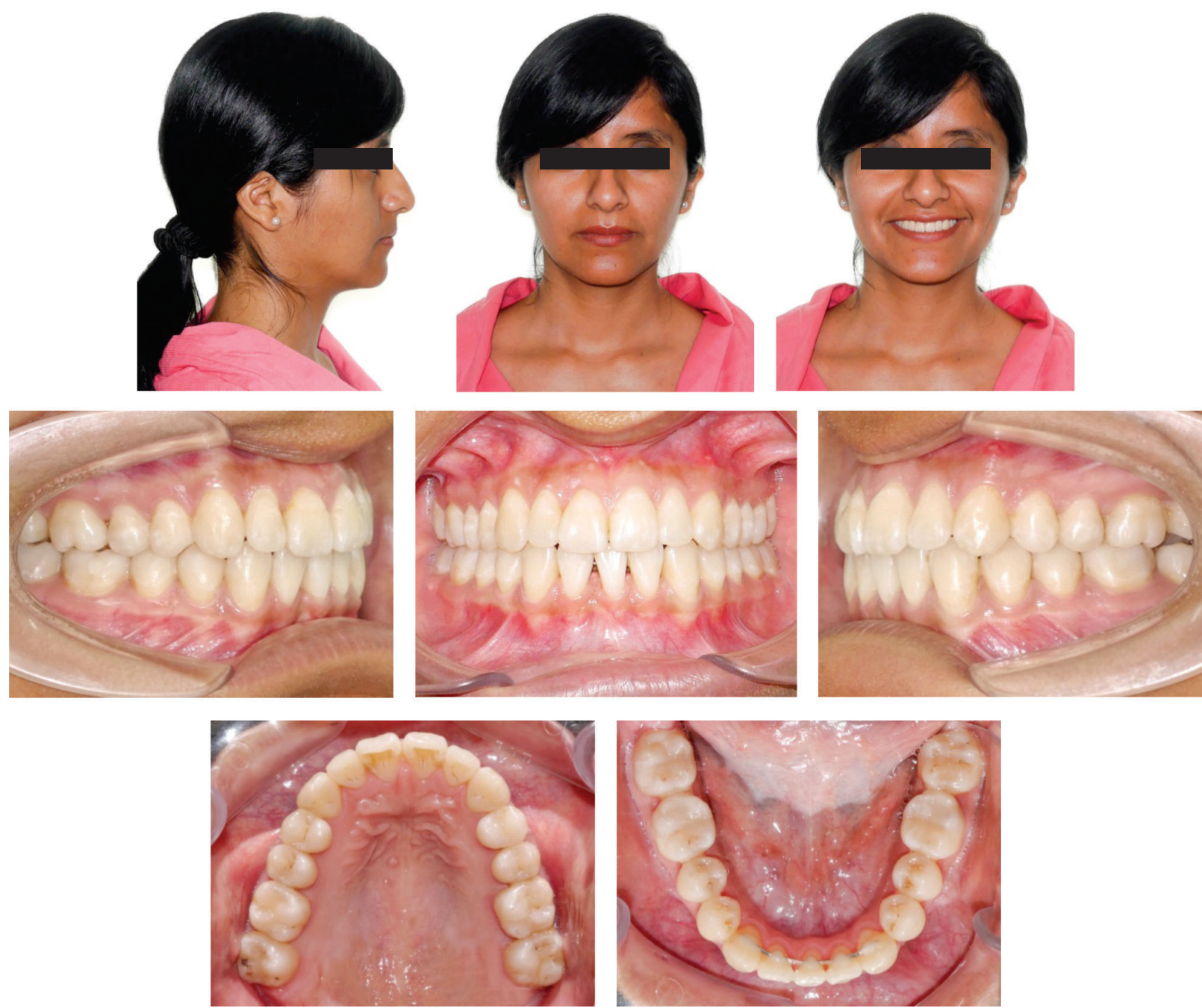

Figura 8. Fotografías extraorales e intraorales postratamiento. 


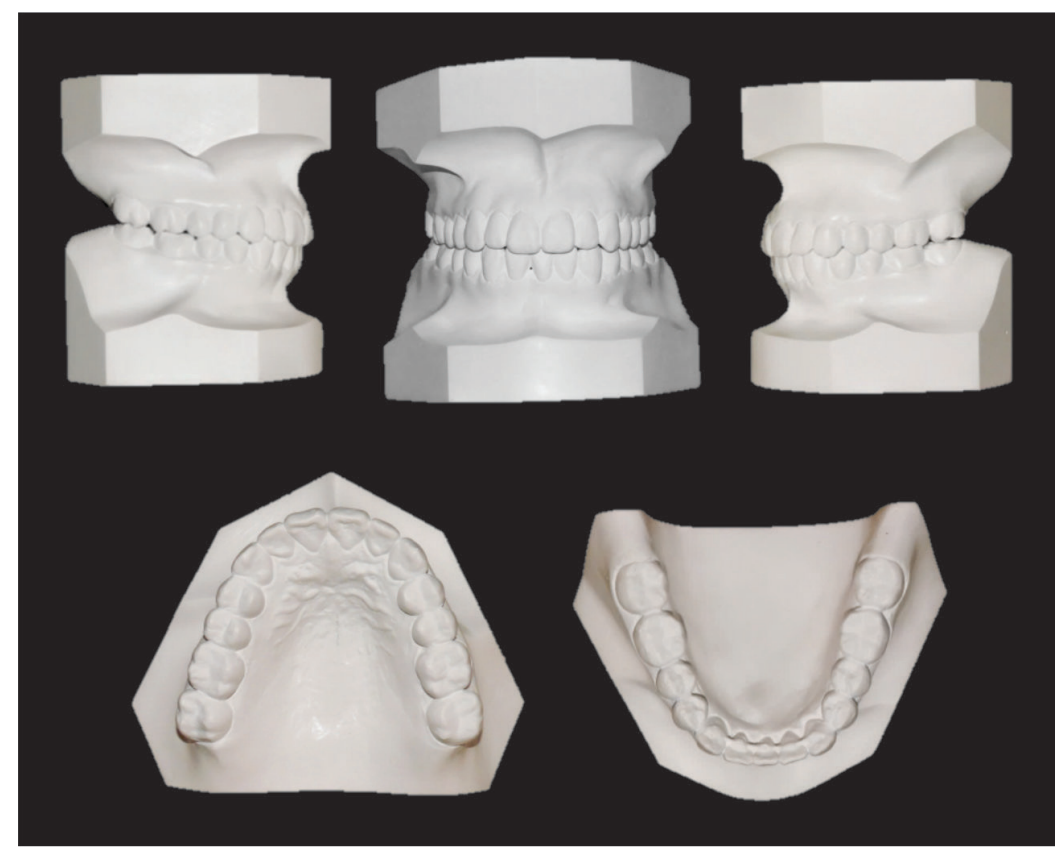

Figura 9. Modelos dentales postratamiento.

superiores e inferiores. Se observa también paralelismo radicular ideal excepto a nivel de los incisivos. Presencia de dilaceración radicular en la pieza 12 (Figura 10).

La evaluación individual y superposición cefalométrica muestran una suave rotación horaria de la mandíbula expresado por el aumento en $1^{\circ}$ del ángulo FMA. Adicionalmente se muestra la proyección de incisivos superiores en $6^{\circ}$ y $1 \mathrm{~mm}$ hacia vestibular. Con respecto a incisivos inferiores se mantuvo su posición e inclinación (Figuras 11 y 12).
Las fotografías extraorales demuestran que el perfil facial total y perfil de los labios se mantuvieron estables. En sonrisa, se exhibe consonancia (Figura 8).

En un control a los 6 meses después del retiro de la aparatología, se verificó la estabilidad de las posiciones y relaciones dentales intra e interarcos. Se observó la estabilidad de las guías funcionales caninas y la guía anterior (Figura 13).

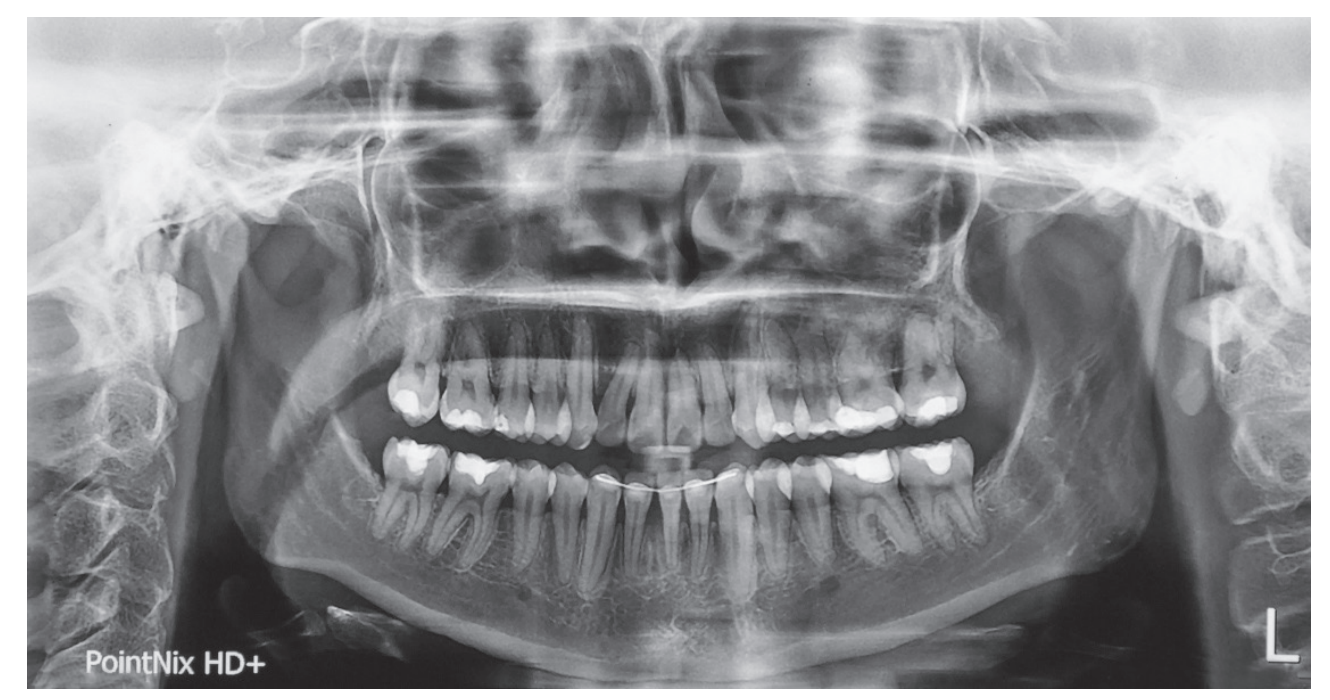

Figura 10. Radiografía panorámica postratamiento. 

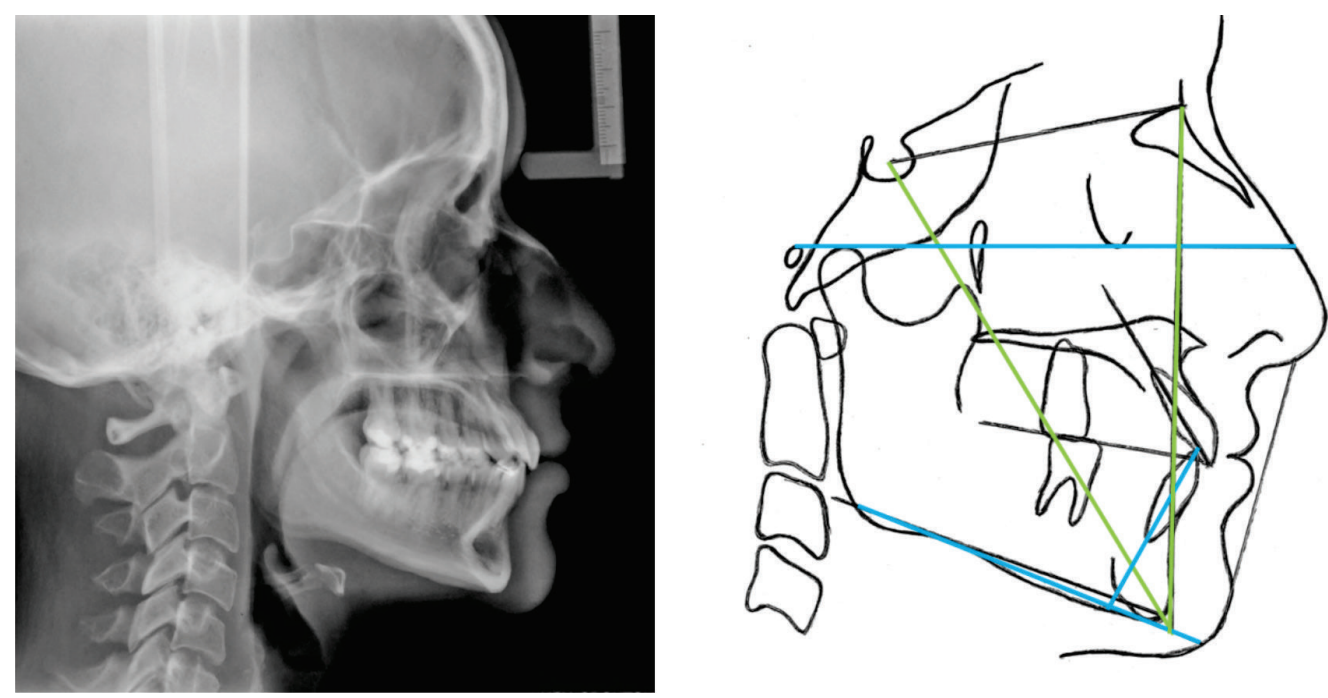

Figura 11. A. Radiografía cefalométrica postratamiento. B. Trazado cefalométrico postratamiento. Las líneas de color negro, azul y verde indican el análisis de Steiner, Tweed y Downs respectivamente.

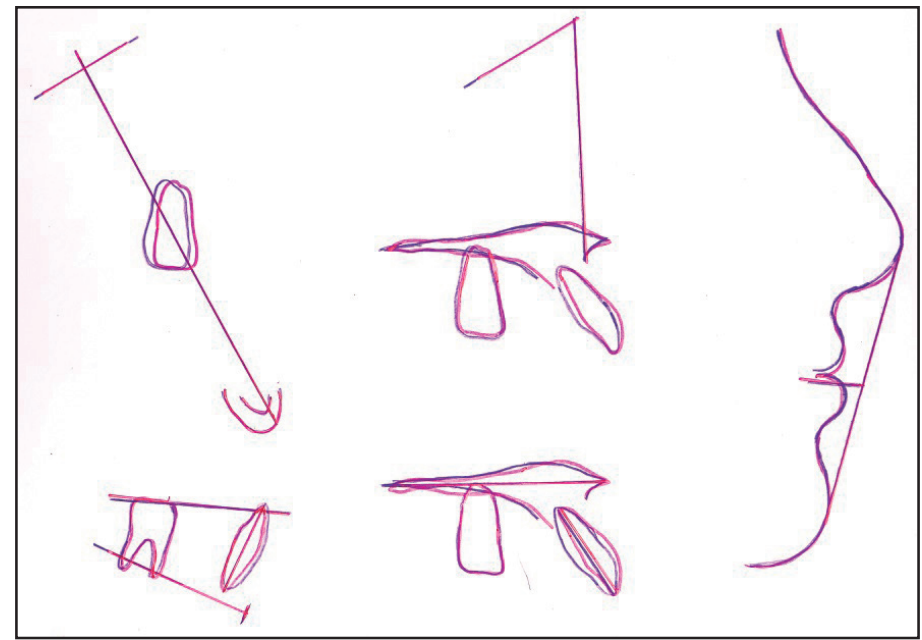

Figura 12. Superposiciones de trazados cefalométricos de pretratamiento (línea de color negro) y de postratamiento (línea de color rojo). Según los cinco campos de Ricketts.
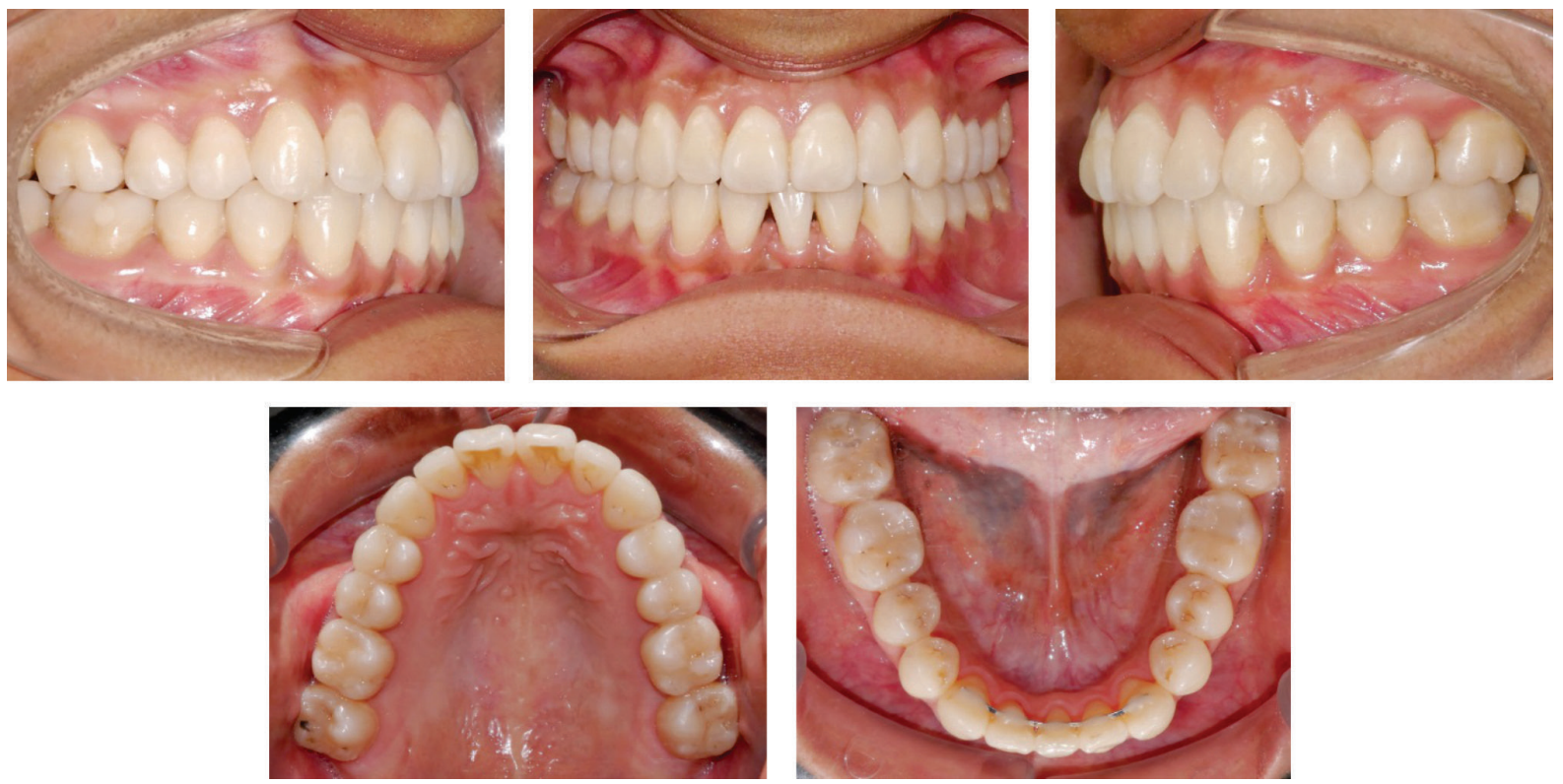

Figura 13. Fotografías intraorales 6 meses postratamiento. 


\section{Discusión}

El tratamiento de ortodoncia con extracción de un incisivo requiere de una rigurosa fase de diagnóstico, ya que el éxito de este tipo de tratamiento depende de las características del caso clínico. El cual debe reunir una serie de requisitos mínimos para un tratamiento predecible y sin sorpresas. Por ello, se hace indispensable realizar un Setup de modelos para determinar principalmente cuáles serán los cambios en las dimensiones de la arcada inferior al extraer un incisivo, la cantidad de desgaste interproximal en incisivos y caninos superiores, y el nuevo overbite y overjet $^{4-11}$.

El tratamiento de ortodoncia con extracción de un incisivo inferior está indicado especialmente para pacientes sin crecimiento, en cuyo motivo de consulta no se incluya el cambio en el perfil de los labios. El patrón esquelético puede ser de Clase I, II y III ${ }^{16-19}$, sin embargo en el análisis clínico intraoral de forma ideal se deben cumplir con cuatro requisitos mínimos: buena relación de intercuspidación de dientes posteriores en Clase I, apiñamiento anteroinferior de $5 \mathrm{~mm}$ a más, discrepancia de Bolton anterior por exceso inferior de $4 \mathrm{~mm}$ a más y un overbite y overjet disminuidos. Adicionalmente, unos incisivos superiores con coronas de forma triangular. Las contraindicaciones para este tipo de tratamiento son el apińamiento superior moderado o severo, incisivos superiores con anomalías de forma y tamaño, candidatos a odontología estética para aumento del ancho mesiodistal e incisivos inferiores con compromiso periodontal ${ }^{2-15}$.

En el presente reporte de caso, la paciente cumplió los requisitos previamente descritos y con el objetivo principal de mantener el perfil de labios, se optó por un tratamiento con extracción de un incisivo inferior; extracción de la pieza 41 por tres razones, de los cuatro incisivos la pieza 41 fue aquella con la posición más protruida, la pieza con mayor compromiso periodontal (recesión gingival) y la pieza con el menor ancho mesiodistal $(5 \mathrm{~mm})$.

Evitar la pérdida de anclaje inferior de molares, premolares y caninos después de la extracción de un incisivo ocasionada por el colapso de la arcada, es una tarea difícil de realizar. En el presente caso se detectó una pérdida de anclaje posterior leve principalmente en el lado derecho. Problema que fue resuelto utilizando arcos multiloops superior e inferior con el protocolo para Clase III ${ }^{20}$.

Para conseguir una adecuada desoclusión canina derecha se recurrieron primero a dos estrategias simples, la de realizar dobleces en los arcos superior e inferior: tip forward de corona de la pieza 13 y tip back de la pieza 43. Sin embargo, fue necesario restaurar la vertiente mesial de la pieza 13 con un sistema de resina universal Filtek Z350 XT (3M ESPE) lo que mejoró la anatomía de la corona, que a su vez permitió una adecuada desoclusión canina en el lado derecho.

Para el presente caso, conseguir un buen overbite y overjet no requirió de un desgaste interproximal mayor a 1,5 $\mathrm{mm}$ de incisivos superiores debido a que estas coronas en su cara palatina presentaron prominencias de esmalte bien desarrolladas.

La contención fija inferior de canino a canino probablemente sea la mejor opción posterior a un tratamiento de ortodoncia con extracción de un incisivo, porque asegura la estabilidad a largo plazo de la posición de los tres incisivos remanentes y evita la aparición de espacios de extracción $^{12,15}$.

La presencia de pérdida de papila o aparición de triángulos negros entre los incisivos inferiores adyacentes a la extracción, es un efecto secundario común del tratamiento con extracción de un incisivo, que se acentúa cuando se tiene coronas de forma triangular. Para prevenir la aparición de triángulos negros, el cierre de espacios después de la extracción del incisivo debe ser por desplazamiento a cuerpo entero, no por inclinación $2,13,14$

\section{Conclusiones}

El tratamiento de ortodoncia fija con extracción de un incisivo inferior es una opción de tratamiento para determinados casos, que reúnan una serie de características previamente descritas en este artículo.

Realizar un Setup de modelos se vuelve un requisito indispensable previo al tratamiento de ortodoncia con extracción de un incisivo inferior.

Es necesario que el paciente conozca los posibles efectos secundarios del tratamiento de ortodoncia con extracción de un incisivo, como la formación de triángulos negros o pérdida de papila entre incisivos inferiores.

Cuando el motivo de consulta del paciente y el tiempo de tratamiento son factores determinantes al momento de elegir la opción de tratamiento, la extracción de un diente versus dos o cuatro dientes en un tratamiento de ortodoncia convencional, la extracción de un incisivo inferior se convierte en una opción más conservadora.

\section{Referencias bibliográficas}

1. Little RM, Wallen TR, Riedel RA. Stability and relapse of mandibular anterior alignment first premolar extraction cases treated by traditional edgewise orthodontics. Am J Orthod. 1981;80(4):349-65.

2. Uribe F, Nanda R. Considerations in mandibular incisor extraction cases. J Clin Orthod. 2009;43(1):45-51.

3. Bahreman AA. Lower incisor extraction in orthodontic treatment. Am J Orthod. 1977;72(5):560-7.

4. Zhylich D, Suri S. Mandibular incisor extraction: a systematic review of an uncommon extraction choice in orthodontic treatment. J Orthod. 2011;38(3):185-95.

5. Vignesh T, Kumar KP, Tamizharasi SK. Mandibular incisor extraction in orthodontics: a systematic review. J Indian Acad Dent Spec Res. 2014;1(1):1-5.

6. Almeida NV, Silveira GS, Pereira DM, Mattos CT, Mucha JN. Interproximal wear versus incisors extraction to solve anterior lower crowding: a systematic review. Dental Press J Orthod. 2015;20(1):66-73. 
7. Matsumoto MA, Romano FL, Ferreira JT, Tanaka S, Morizono EN. Lower incisor extraction: an orthodontic treatment option. Dental Press J Orthod. 2010;15(6):143-61.

8. Lima CM, Lacet E, Marques CR. Extração de incisivo inferior: uma opção terapéutica. R Dental Press Ortodon Ortop Facial. 2005;10(4):47-59.

9. Lessa TL, Arruda E, Assad RA, Sfier F, Dabul A. Extração de incisivo inferior em ortodontia. Revista Gestão \& Saúde. 2013;9(1):1-11.

10. Kokich VG, Shapiro PA. Lower incisor extraction in orthodontic treatment. Four clinical reports. Angle Orthod. 1984;54(2):139-53.

11. Valinoti JR. Mandibular incisor extraction therapy. Am J Orthod Dentofacial Orthop. 1994;105(2):107-16.

12. Canut JA. Mandibular incisor extraction: indications and long-term evaluation. Eur J Orthod. 1996;18(5):485-9.

13. Uribe F, Holliday B, Nanda R. Incidence of open gingival embrasures after mandibular incisor extractions: a clinical phographic evaluation. Am J Orthod Dentofacial Orthop. 2011;139(1):49-54.

14. Rachala MR, Aileni KR, Dasari AK, Sinojiya J. Biomechanical considerations in mandibular incisor extraction cases. Int J Orthod Milwaukee. 2015;26(1):47-51.
15. Riedel RA, Little RM, Bui TD. Mandibular incisor extraction postretention evaluation of stability and relapse. Angle Orthod. 1992;62(2):103-16.

16. Guo Y, Han X, Xu H, Ai D, Zeng H, Bai D. Morphological characteristics influencing the orthodontic extraction strategies for Angle's class II division 1 malocclusions. Prog Orthod. 2014;15(1):44.

17. Klein DJ. The mandibular central incisor, an extraction option. Am J Orthod Dentofacial Orthop. 1997;111(3):253-9.

18. Faerovig E, Zachrisson BU. Effects of mandibular incisor extraction on anterior occlusion in adults with class III malocclusion and reduced overbite. Am J Orthod Dentofacial Orthop. 1999;115(2):113-24.

19. Lertnimulchai S, Manosudprasit M. One lower incisor extraction in orthodontic treatment: correction of having mandibular anterior tooth size excess. KDJ. 2000;3(1):121-7.

20. Kim YH, Han UK. The versatility and effectiveness of the multiloop edgewise archwire (MEAW) in treatment of various malocclusions. World J Orthod. 2001;2(3):20818. 